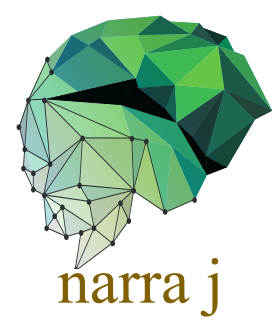

Short Communication

\title{
Investigation of the role of sleep quality and sleep duration on fasting blood glucose level in acute ischemic stroke patients: $A$ preliminary study
}

\author{
Riza P. Putra1,2 and Wardah R. Islamiyah ${ }^{1,2^{*}}$ \\ ${ }^{1}$ Department of Neurology, Faculty of Medicine, Universitas Airlangga, Surabaya, Indonesia; ${ }^{2}$ Dr. Soetomo Hospital, \\ Surabaya, Indonesia \\ *Corresponding author: wardah-r-i@fk.unair.ac.id
}

\begin{abstract}
The aim of this study was to evaluate the association between the sleep quality and sleep duration with the level of fasting blood glucose in patients with acute ischemic stroke. A cross-sectional study was conducted among acute ischemic stroke patients admitted to Dr. Soetomo Hospital and Universitas Airlangga Hospital from May to October 2021. Patients who met the inclusion were enrolled. To assess the sleep quality, Pittsburgh Sleeps Quality Index (PSQI) was applied. To determine sleep duration during the nocturnal period, Fitbit Charge 2 HR device was used. Evaluation of plausible confounders was assessed through physical examination, interviews, and a set of validated questionnaires. A total of 30 acute ischemic stroke patients were included in this study. The mean age of the participants was $56.40 \pm 9.77$ years and $60 \%$ were males. There were $63.3 \%$ patients classified as mild stroke and $36.7 \%$ moderate-severe stroke. The average PQSI score was $5.57 \pm 2.59$, while the average sleep duration was $319.8 \pm 108.7$ minutes. The average fasting blood glucose level was $89.03 \pm 14.71 \mathrm{mg} / \mathrm{dL}$. Significant positive correlation was obtained between sleep duration and fasting blood glucose level $(\mathrm{r}=0.533 ; \mathrm{p}=0.002)$. Similarly, there was no statistically significant correlation between sleep quality with the level of fasting blood glucose ( $\mathrm{r}=-0.167$; $\mathrm{p}=0.377$ ). To the best of our knowledge, this is the first study assessing the correlation between sleep quality and sleep duration with the level of fasting blood glucose levels in patients with acute ischemic stroke. This study therefore might be of great interest to provide insights on the importance of sleep management in acute ischemic stroke patients.
\end{abstract}

Keywords: Acute ischemic stroke, diabetes mellitus, fasting blood glucose, prediabetes, sleep quality

\section{Introduction}

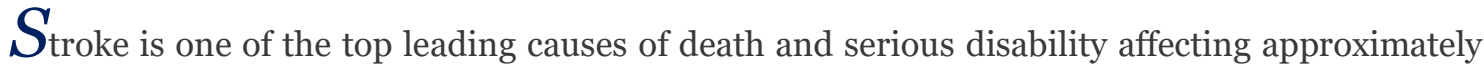
13.7 million people each year worldwide [1]. In the US, approximately 795,000 cases reported annually with 610,000 and 185,000 are recorded as the first-attack and recurrent strokes, respectively [2]. Of the total stroke incidents, $87 \%$ are ischemic stroke whereas the remaining $10 \%$ and $3 \%$ are intracerebral and subarachnoid hemorrhage, respectively [2].

Type 2 diabetes mellitus (DM) and prediabetes are considered the major risk factors for ischemic stroke. DM doubles the risk for ischemic and prediabetes (impaired fasting blood 
glucose and/or impaired glucose tolerance) serves as a potential risk factor for DM. More than two-thirds of prediabetes patients develop DM [3]. Individuals with prediabetes possess a 20\%greater risk of stroke compared to those with normal blood glucose [4]. Prediabetes is also associated with a double-increased risk of stroke recurrence in nondiabetic patients with transient ischemic attack (TIA) or ischemic stroke [5]. Various types of sleep disorders such as poor sleep quality and short sleep duration have been reported to increase the risk for both prediabetes and DM through interference with glucose homeostasis regulation [6]. Poor sleep quality is associated with a two-fold increase in the risk of prediabetes [7], while insufficient sleep duration is correlated with the occurrence of DM or prediabetes by two-fold $[5,8]$.

Reduction in sleep quality and alteration in sleep duration are major complaints in more than $50 \%$ of stroke survivors $[9,10]$. Poor quality of sleep and inappropriate sleep duration increases the risk of recurrent stroke through the alteration of glucose metabolism, observed by the increase in blood glucose level [5-8]. Therefore, determining the association between sleep quality and sleep duration with risk of prediabetes, increased risk of ischemic stroke, as well as recurrent ischemic stroke is highly important. Early evaluation on sleep quality and sleep duration may reduce the number of ischemic stroke recurrences, particularly in developing countries. Hence, this study sought to evaluate the association between sleep quality and sleep duration with fasting blood glucose level in acute ischemic stroke patients, as well as their potential confounders.

\section{Methods}

\section{Study design and setting}

A cross-sectional study was conducted among acute ischemic stroke patients admitted to Dr. Soetomo Hospital and Universitas Airlangga Hospital from May to October 2021. Patients who met the inclusion criteria were enrolled after being previously informed of the study and provided written consent. The inclusion criteria were acute ischemic stroke patients $>20$ years of age; being within 1-3 days from the onset of ischemic stroke; having Glasgow Coma Scale of E4M5V6; and available for body mass index (BMI) measurement. Patients with or a history of DM, hepatic cirrhosis, chronic kidney failure and autoimmune disease, pregnant and those under antidiabetic and steroid drugs treatments were excluded. Demographic data and confounders data were collected through the physical examination and a face-to-face interview.

\section{Assessment of sleep quality, sleep duration, and fasting blood glucose level}

On day 7 of hospital admission, the sleep quality, sleep duration, and fasting blood glucose level were measured. The Pittsburgh Sleep Quality Index (PSQI) was used to evaluate the sleep quality [11]. The PSQI consists of 19-self-rating items measure several aspects including sleep onset latency, sleep fragmentation, total wake time, sleep efficiency, and sleep disruptive events and the total scores ranging from $0-21$. To assess the sleep duration during nocturnal period, Fitbit Charge 2 Heart Rate (Fitbit, Inc. San Fransisco, CA, US) was installed on the patient's unimpaired wrist for four days, starting from day 3-6 from the stroke onset. The device was continuously worn by patients every day for 12-hours period (from $6 \mathrm{pm}$ to $6 \mathrm{am}$ ). To measure the fasting blood glucose level, the venous blood was collected between 6-7 pm after 8-hours fasting and the fasting blood glucose level was tested using an enzyme hexokinase (HK) method with Cobas C 501 (Roche, Switzerland) following the manufacturer protocol.

\section{Assessment of potential confounders}

Face-to-face interviews, assisting with a set of validated questionnaires, were conducted to collect information on potential confounders affecting the level of fasting blood glucose. These factors included gender, age, BMI, alcohol consumption, smoking status, history of hypertension, family history of diabetes, and stroke severity. For statistical purpose, the age was grouped into two groups ( $<45$ years and $\geq 45$ years) and the BMI was classified as normal and overweight. The level of stroke severity was determined according to the National Institute of Health Stroke Scale (NIHSS). NIHSS score $<5$ was classified as mild stroke while score $\geq 5$ as moderate-severe stroke. 


\section{Statistical analysis}

The Mann-Whitney test was used to assess the effect of potential confounders (age, BMI, smoking status, history of hypertension, family history of diabetes, and stroke severity) on fasting blood glucose levels in patients with acute ischemic stroke. The Spearman correlation was employed to identify the correlation between sleep quality score and sleep duration with the fasting blood glucose levels. All analyses were performed by means of IBM SPSS software version 26.0.

\section{Results}

\section{General characteristics of participants}

A total of 30 acute ischemic stroke patients were enrolled. The baseline characteristics of the patients are presented in Table 1. The patients ages ranged from 37 to 83 years (mean 56.4 \pm 9.7 years). Of the total 30 patients, 18 (60.0\%) were male and the mean of BMI was $24.0 \pm 3.5$ $\mathrm{kg} / \mathrm{m}^{2}$. There were no patients with a history of alcohol consumption. There were 12 (40.0\%) smokers and 2 (6.7\%) ex-smokers. The number of patients with a history of hypertension was $22(73.3 \%)$, while those with a family history of diabetes was 10 (33.3\%). Patients with mild and moderate-severe stroke were $19(63.3 \%)$ and 11 (36.7\%), respectively.

Table 1. Characteristics of the participants $(n=30)$

\begin{tabular}{|c|c|}
\hline Characteristics & Frequency (\%) \\
\hline \multicolumn{2}{|l|}{ Gender } \\
\hline Male & $18(60.0)$ \\
\hline Female & $12(40.0)$ \\
\hline \multicolumn{2}{|l|}{ Age (year), mean $\pm \mathrm{SD}$} \\
\hline$<45$ years & $3(10.0)$ \\
\hline$\geq 45$ years & $27(90.0)$ \\
\hline \multicolumn{2}{|l|}{ Body mass index (BMI) $\left(\mathrm{kg} / \mathrm{m}^{2}\right)$} \\
\hline Normal & $17(56.7)$ \\
\hline Overweight & $13(43.3)$ \\
\hline \multicolumn{2}{|l|}{ Alcohol consumption } \\
\hline Yes & $\mathrm{o}(0.0)$ \\
\hline No & $30(100.0)$ \\
\hline \multicolumn{2}{|l|}{ Smoking status } \\
\hline Smoker & $12(40.0)$ \\
\hline Ex-smoker & $2(6.7)$ \\
\hline Non-smoker & $16(53.3)$ \\
\hline \multicolumn{2}{|l|}{ History of hypertension } \\
\hline Yes & $22(73 \cdot 3)$ \\
\hline No & $8(26.7)$ \\
\hline \multicolumn{2}{|l|}{ Family history of diabetes } \\
\hline Yes & $10(33.3)$ \\
\hline No & $20(66.7)$ \\
\hline \multicolumn{2}{|l|}{ Stroke severity } \\
\hline Mild & $19(63 \cdot 3)$ \\
\hline Moderate-severe & $11(36.7)$ \\
\hline Sleep quality, mean \pm SD & $5 \cdot 57 \pm 2.59$ \\
\hline Average night-sleep duration, mean $\pm \mathrm{SD}$ & $319.83 \pm 108.78$ \\
\hline Fasting blood glucose level, mean \pm SD & $89.03 \pm 14.71$ \\
\hline
\end{tabular}

SD: standard deviation

\section{Sleep quality, duration, and blood glucose level in acute ischemic stroke patients}

Sleep quality was measured using PSQI; the lowest PQSI score was 3 while the highest was 11 (median 4.50; mean 5.57 \pm 2.59 ). The shortest of total sleep duration recorded in nocturnal period was 144 minutes, while the longest was 588 minutes (median 323.00; mean $319.83 \pm 108.78)$. The median and the mean of the blood glucose level under were 86.00 and $89.03 \mathrm{mg} / \mathrm{dL}$, respectively; ranged between $65 \mathrm{mg} / \mathrm{dL}$ and $129 \mathrm{mg} / \mathrm{dL}$ (Table 1). 
The association between confounder variables and the level of fasting blood glucose level

Our data suggested that the mean blood glucose level had no association with patients' age, BMI, smoking status, history of hypertension, family history of diabetes, and stroke severity (Table 2). Although the mean blood glucose level was higher in patients under 45 years of age, in those who were overweight, those who had a history of hypertension and family history of diabetes, as well as in patients with moderate-severe stroke compared to those of patients over 45 years, who had normal BMI, had no a history of hypertension, had no family history of diabetes, and those with mild stroke, respectively, the levels were not significantly different.

Table 2. The association between variables and the level of fasting blood glucose $(n=30)$

\begin{tabular}{lll}
\hline Variables & Mean \pm standard deviation $(\mathrm{mg} / \mathrm{dL})$ & $\mathrm{p}$-value \\
\hline Gender & $88.39 \pm 14.74$ & 0.750 \\
$\quad$ Male & $90.00 \pm 15.26$ & \\
$\quad$ Female & $91.00 \pm 2.64$ & \\
Age (year) & $88.81 \pm 15 \cdot 50$ & \\
$\quad<45$ & & \\
$\geq 45$ & $87.24 \pm 14.11$ & 0.402 \\
Body mass index (BMI) $\left(\mathrm{kg} / \mathrm{m}^{2}\right)$ & $91.38 \pm 15 \cdot 71$ & \\
$\quad$ Normal & $89.03 \pm 14.71$ & 0.750 \\
$\quad$ Overweight & $83.00 \pm 8.48$ & \\
Smoking status & $89.56 \pm 16.21$ & \\
$\quad$ Smokers & $89.41 \pm 13.13$ & 0.411 \\
$\quad$ Ex-smokers & $88.00 \pm 19.44$ & \\
$\quad$ Non-smokers & & \\
History of hypertension & $90.25 \pm 15.22$ & 0.567 \\
$\quad$ Yes & $86.60 \pm 14.09$ & \\
No & & \\
Family history of diabetes & $88.68 \pm 14.89$ & 0.750 \\
$\quad$ Yes & $89.64 \pm 15.10$ & \\
$\quad$ No &
\end{tabular}

The correlation of sleep quality and sleep duration with fasting blood glucose levels

The results of Spearman correlation suggested that there was no correlation between the sleep quality and the fasting blood glucose level in acute ischemic stroke patients $(r=-0.167 ; p=0.377)$. Nevertheless, there was a significant positive correlation between sleep duration and blood glucose level among patients with acute ischemic stroke $(\mathrm{r}=0.533 ; \mathrm{p}=0.002)$.

\section{Discussion}

The current study was conducted to evaluate the association between sleep quality and sleep duration, as well as several confounding factors with the level of fasting blood glucose in acute ischemic stroke patients. Sleep quality is defined as an individual's satisfaction with the sleep experience, integrating aspects of sleep initiation, maintenance, quantity, and refreshment upon awakening. In addition, sleep quality is also recognized as subjective and objective sleep parameters associated with sleep disruption severity, such as sleep onset latency, total wakefulness, sleep efficiency, number of awakenings, and arousal during a nocturnal sleep period [12]. Sleep duration is the total amount of sleep obtained either during the nocturnal or over a 24-hour sleep period [13]. Sleep disruption has been the major complaint among acute ischemic stroke patients, subsequently leading to the alteration in glucose metabolism [5-9, 14]. Our data suggested no significant correlation between the sleep quality with fasting blood glucose levels.

This result differed from that reported in the previous study, indicated a significantly higher prevalence of impaired blood glucose levels in individuals with poor sleep quality and short sleep duration compared to those with good sleep quality and having 6-8 hours of sleep duration [15]. A possible assumption of such insignificantly negative correlation in our current 
study is that there was probably some serious alteration in the patients' sleep quality during their hospitalization period due to the acute ischemic stroke incidents, which could not be assessed using the PSQI questionnaire.

Sleep duration is classified into 3 categories: short ( $<6$ hours), normal (6-8 hours), and long ( $>8$ hours) [16]. However, the time of sleep needed between individual were depend on the age, the older the age, the shorter the time of sleep needed [13]. Our results suggested a positive correlation between nocturnal sleep duration and fasting blood glucose levels, indicating that the shorter the sleep duration, the lower the level of fasting blood glucose. This finding is contrary to our study hypothesis and the findings of several former studies, suggesting that short sleep duration is associated with the risk for elevated blood glucose levels and hyperglycemia $[16,17]$. Some plausible explanations for this are the disrupted elements of the sleep stages involved in the present study were different from those of previous studies [16, 17]. Brain glucose uptake and muscle glucose utilization during the awakening stage might result in a decreased level of plasma glucose at the end of the fasting period since glucose consumption during rapid eye movement (REM) sleep and awakening phase is reportedly higher compared to that during non-rapid eye movement (NREM) sleep [18]. Glucose metabolism is also influenced by other sleep disturbances such as sleep-disordered breathing (SDB) which might occur as a result of acute ischemic stroke incidents [19, 20]. Taken together these could be responsible for such different findings.

\section{Strengths and limitations}

In this study, we used highly specific study subjects who were acute ischemic stroke patients. To the best of our knowledge, this is the first study assessing the role of sleep quality and sleep duration with the level of fasting blood glucose in acute ischemic stroke group. This study however possessed several limitations. The number of patients is relatively small. This study is unable to control certain sleep disturbances affecting glucose metabolism such as sleepdisordered breathing (SDB). A specific assessment regarding the sleep stages and the control of the amount of calorie uptake by the patients during the investigation was not also possible to be conducted. Therefore, studies are recommended to further evaluate factors associated with blood glucose levels in patients with acute ischemic stroke for a more definitive conclusion including to focus on specific severity of acute ischemic stroke.

\section{Conclusion}

Our study suggested no significant correlation between sleep quality and the fasting blood glucose level in acute ischemic stroke. However, a positive correlation between sleep duration and the level of blood glucose was observed. This study provides basic information and insights on the importance of sleep management in acute ischemic stroke patients. Since our study possessed several limitations, further evaluations, particularly on the association between each of sleep stages as well as sleep quality parameters and the blood glucose level are strongly recommended, it could enable as independent assessment between sleep disturbances and blood glucose levels in critical patients.

\section{Ethics approval}

The protocol of study was approved by the Research Ethics Committee of Dr. Soetomo Hospital (0190/KEPK/V/2021) and Universitas Airlangga Hospital (183/KEP/2021). Acknowledgments

\section{Acknowledgments}

We acknowledge Universitas Airlangga and the staff of Dr. Soetomo Hospital and Universitas Airlangga Hospital in Surabaya.

\section{Conflict of interest}

The authors declare that they have no competing interests. 


\section{Funding}

This work is received partial funding of Lembaga Pengelola Dana Pendidikan (LPDP).

\section{Underlying data}

Derived data supporting the findings of this study are available from the first author on request.

\section{How to cite}

Putra RP and Islamiyah WR. Investigation of the role of sleep quality and sleep duration on fasting blood glucose level in acute ischemic stroke patients: Results from a preliminary study. Narra J 2021; 1(3): e59 - http://doi.org/10.52225/narra.v1i3.59.

\section{References}

1. Lindsay MP, Norrving B, Sacco RL, et al. World Stroke Organization (WSO): global stroke fact sheet 2019. In: SAGE Publications Sage UK: London, England; 2019.

2. Virani SS, Alonso A, Aparicio HJ, et al. Heart disease and stroke statistics-2021 update: a report from the American Heart Association. Circulation 2021; 143(8):e254-e743.

3. Mijajlović MD, Aleksić VM, Šternić NM, et al. Role of prediabetes in stroke. Neuropsychiatr Dis Treat 2017; 13:259

4. Lee M, Saver JL, Hong K-S, et al. Effect of pre-diabetes on future risk of stroke: meta-analysis. BMJ 2012; 344:e3564.

5. Fonville $\mathrm{S}$, Zandbergen AA, Koudstaal PJ, et al. Prediabetes in patients with stroke or transient ischemic attack: prevalence, risk and clinical management. Cerebrovasc Dis 2014; 37(6):393-400.

6. Briançon-Marjollet A, Weiszenstein M, Henri $M$, et al. The impact of sleep disorders on glucose metabolism: endocrine and molecular mechanisms. Diabetol Metab Synd 2015; 7(1):1-16.

7. Ghorbani A, Esmailzadehha N, Mohammadpoorasl A, et al. Association of sleep quality and waking time with prediabetes: The qazvin metabolic diseases study, Iran. Sleep Disord 2015;2015:480742.

8. Engeda J, Mezuk B, Ratliff S, et al. Association between duration and quality of sleep and the risk of pre-diabetes: evidence from NHANES. Diabetic Med 2013; 30(6):676-680.

9. Ferre A, Ribó M, Rodriguez-Luna D, et al. Strokes and their relationship with sleep and sleep disorders. Neurologia (English Edition) 2013; 28(2):103-118.

10. Khot SP, Morgenstern LB. Sleep and stroke. Stroke 2019; 50(6):1612-1617.

11. Buysse DJ, Reynolds CF, 3rd, Monk TH, et al. The Pittsburgh Sleep Quality Index: a new instrument for psychiatric practice and research. Psychiatry Res 1989; 28(2):193-213.

12. Kline C. Sleep quality. Encyclopedia of behavioral medicine 2013:1811-1813.

13. Chaput J-P, Dutil C, Sampasa-Kanyinga H. Sleeping hours: what is the ideal number and how does age impact this? Nat Sci Sleep 2018; 10:421.

14. Hasan F, Gordon C, Wu D, et al. Dynamic prevalence of sleep disorders following stroke or transient ischemic attack: systematic review and meta-analysis. Stroke 2021; 52(2):655-663.

15. Lou P, Chen P, Zhang L, et al. Interaction of sleep quality and sleep duration on impaired fasting glucose: a population-based cross-sectional survey in China. BMJ Open 2014; 4(3):e004436.

16. Rafalson L, Donahue RP, Stranges S, et al. Short sleep duration is associated with the development of impaired fasting glucose: the Western New York Health Study. Annals Epidemiol 2010; 20(12):883-889.

17. DePietro RH, Knutson KL, Spampinato L, et al. Association between inpatient sleep loss and hyperglycemia of hospitalization. Diabetes Care 2017; 40(2):188-193.

18. Sharma S, Kavuru M. Sleep and metabolism: an overview. Int J Endocrinol. 2010; 2010: 270832

19. Stevens D, Martins RT, Mukherjee S, et al. Post-stroke sleep-disordered breathing-pathophysiology and therapy options. Front Surg 2018; 5: 9.

20. Blissitt PA. Sleep-disordered breathing after stroke: nursing implications. Stroke 2017; 48(3):e81-e84. 